\title{
Hydro-seismic-acoustical monitoring of submarine earthquakes preparation: observations and analysis
}

\author{
E. V. Sasorova ${ }^{1}$, B. W. Levin ${ }^{2}$, and V. E. Morozov ${ }^{1}$ \\ ${ }^{1}$ Shirshov Institute of Oceanology of Russian Academy of Sciences, Moscow, Russia \\ ${ }^{2}$ Institute of Marine Geology and Geophysics FEB RAS, Yuzhno-Sakhalinsk, Russia
}

Received: 14 May 2007 - Revised: 30 October 2007 - Accepted: 10 November 2007 - Published: 2 January 2008

\begin{abstract}
The results of laboratory experiments on rock sample destruction and the observation data obtained from several series of the hydro-acoustic observations in which the researchers succeeded to register the signals in the critical stage of the earthquake (EQ) preparation were compared. According to theoretical research (Alekseev et al., 2001) two distinct dilatant zones occur in the EQ preparation stage. The first one is located around the source and the second one represents the near-surface dilatant zone. Only high-frequency seismic-acoustic signals (SAS) radiated from the near-surface dilatant zone do not attenuate completely on the passage through a solid medium. Parameters of the SAS such as the source depth under the ocean floor, frequency maximum and the signal power level were estimated. It was shown that the critical stage of the EQ preparation continues several tens hours and this process has a hierarchical nature. At first the micro-ruptures are formed over a large area. Then the high frequency radiation begins to decrease, the SAS emission area begins to shrink and the micro-earthquakes occur in the area surrounding the epicenter. The obtained results are in close agreement with the theoretical conception about the evolution of the SAS in the surface dilatant zone and with the results of laboratory experiments.
\end{abstract}

\section{Introduction}

The EQ preparation zones are characterized by increased value of the shear and tension stress in the Earth's crust and the process of the micro-crack appearance. Such zones are named the dilatant zones. An increase of seismic emission activity during the period of EQ preparation and a decrease of this process immediately after the event has been observed (Michihiro et al., 1989; Sobolev, 1993). Partial destruction

Correspondence to: E. V. Sasorova

(sasorova_lena@mail.ru) of solid rocks leads to the acoustic wave generation, whose periods depend on the size of the destruction zones. The attenuation of the acoustic signal increases with the increment of its frequency. Also the SAS decay quickly with the distance (Clay and Medwin, 1977; Sheriff and Geldart, 1995). The attenuation of the acoustic signals at the frequency of $100 \mathrm{~Hz}$ in water layer is 100 times smaller than that in a hard rock and 10000 times smaller than that in the sediments.

Thus a seismic-acoustic emission radiated from a crust quake source, which is located under the sea bottom, generates a hydro-acoustic (HA) signal in water, where this signal may propagate far due to weak attenuation of the signal in incompressible water. In a water layer, the HA signals can be recorded by hydro acoustic receivers. The acoustic signals, which are generated by micro-cracks in the near-focus zone of a future EQ, may be detected in the water only for shallow EQ's (less than 10-15 km). If the EQ source is located deeper, the SAS will decay completely in the solid layers. The numerical simulation of the preliminary fracture process in accordance with Shleicher-Nadai criterion was carried out in theoretical research (Alekseev et al., 2001).

The space-time density of the cracks is used as an integral earthquake precursor in the area of an earthquake preparation. The numerical simulation detected the distinct dilatant zones, where the rupture process affected by stress increase turns into the non-linear stage. An irreversible process of the medium state change arises in these zones. Two distinct dilatant zones were detected in the EQ preparation stage. The first one is located around the source and the second one represents the near-surface dilatant zone, whose horizontal extension may be up to $500 \mathrm{~km}$ and its depth is estimated as about $5 \mathrm{~km}$ (Fig. 1). The dilatant zones are marked by an increase of the micro-crack density and a crack system evolution (crack sizes grow progressively, and their number decreases). Only the high-frequency SAS radiated from near-surface dilatant zone do not attenuated completely on the passage through a solid medium.

Published by Copernicus Publications on behalf of the European Geosciences Union. 


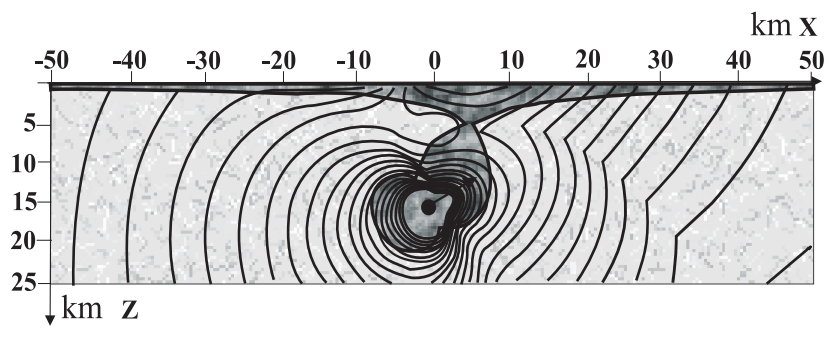

Fig. 1. Two specifically dilatant zones (dark gray areas) occur in the earthquake preparation stage. The first one locate around the focus (small black circuit) and the second one is the near-surface dilatant zone. The figure was adopted from work (Alekseev et al., 2001).

The appearance of acoustic signals preceding the rock sample destruction was studied in the laboratory experiments in the works of numerous authors. Here we take into consideration series of the works (Smirnov et al., 1995; Ponomarev et al., 1997) because in these works, a loading regime with negative feedback was used (the rate of the stress variation decreased with increasing acoustic activity). This allowed to extend the micro-rupture process stage and to detect the micro-destructions along the body of the rock sample as a function of time. Micro-destruction signals have small amplitude, significantly high frequency and these signals decay very fast. Initially, the micro-destruction sources are located chaotically in the body of the rock sample. Then microdestruction clusterization arises in time, a linear size of the destructions increases and the destructions begin to concentrate in a zone of future macro-destruction.

A main objective of our study was to trace the evolution process of seismic-acoustic emission in the critical stage from on EQ preparation area. It has been observed that the high frequency SAS could not be registered by land-based seismic stations because of fast decaying of such signals in solid medium and in the sediments. The acoustic receivers located in the water layer can register seismic events with very wide spectrum of the energy ranges (from large EQ up to micro earthquakes and micro-rupture). In contrast networks of ground-based seismic stations can register only the EQ's. Only hydro acoustic receivers can register micro earthquakes and micro-ruptures. We call seismic event with signal duration from one second up to tens seconds microearthquake (MEQ), while the event with signal duration less than 100 milliseconds are called micro-rupture (MRP).

\section{Analysis of observation data}

Seismic signals originated in solid medium reach the ocean floor and, after transforming on the water/bottom boundary, propagate in the water layer. Only compression waves $(P)$ are propagating in water, the transverse waves $(S)$ convert at the water/bottom boundary into compression waves $(P S)$ and then both waves propagate in the water layer with the same velocity. The difference in arrival times of these waves $\left(T_{p p s}\right)$ to the receiving array enables us to estimate the depth of the seismic event focus under the bottom. It is possible to estimate the following parameters of seismic events by using the HA records (Sasorova et al., 2005): the depth of the source under ocean floor $\left(H_{S}\right)$; the maximum signal frequency $\left(F_{\max }\right)$ and a linear dimension of the source $\left(L_{S}\right)$ as a function of $F_{\max }$ (Sassorova et al., 2001); the duration of the HA signal $\left(t_{S}\right)$ which can specify the power level (magnitude $\mathrm{Ml}$ ) of the SAS registered by HA receivers. The Brocher expression $\mathrm{Ml}=2.30+\lg t_{s}$ (Brocher, 1983) can be used for events with duration above tens seconds and more, and we used the calibration graph proposed by (Soloviev and Kovachev, 1996) if the signal duration was less than $40 \mathrm{~s}$. If the MEQ focus is located not far from the HA receiver (no farther than $100 \mathrm{~km}$ ) then the structure of seismic signal remains completely on the HA record ("close" MEQ). The farther MEQ focus is located from the HA receiver, the more significant are the seismic signal distortions, and the HA record of seismic signal can attain a trapezoidal shape ("remote" MEQ).

Then in the frame of this work, we carried out analysis of data obtained from several series of the HA observations in which the researchers succeeded to register the SAS signals from the surface dilatant zone in the EQ preparation stage. The comparison of HA data with ground seismic observations was produced on the basis of the Kamchatka and Sakhalin Regional Earthquake Catalogs and the World Electronic Catalog produced by of the USGS (NEIC).

The first data series was obtained from bottom selfcontained system (BSAS), which was deployed in the near bottom water layer. One all-around looking hydrophone was used. The continuous record of the HA signals (duration up to $24 \mathrm{~h}$ ) in two frequency ranges ( 2 to $100 \mathrm{~Hz}$ and 100 to $1400 \mathrm{~Hz}$ ) was produced. The BSAS was used in high-activity seismic area (Pacific Kamchatka coastline, Pacific Kuril Islands coastline and Okhotsk sea).

The HA records which were coincident with the seismic events or preceded them (by no more than 3-4h) were extracted for the subsequent analysis. Thus several records were selected. The EQ's on these records were preceded by the series of the MEQ's. One of the selected records with duration $15 \mathrm{~h}$ and $20 \mathrm{~min}$ contains one EQ (date - 30.03.1988, local time $-23: 26: 27.9 ; K_{S}=9.2$, depth $-34 \mathrm{~km}$, coord.: $54.510 \mathrm{~N}, 161.620 \mathrm{~W}$ ) and a time sequence of $60 \mathrm{MEQ}$ 's. The MEQ duration varies from 0.8 to $103 \mathrm{~s}$ but the duration of the overwhelming majority of the MEQ's was 3-4 s. The $F_{\max }$ value for MEQ varies from 3 to $100 \mathrm{~Hz}$. A clearly expressed tendency to MEQ clusterization was evident; the density of events in time unit before EQ was increased as well as the number of MEQ's with duration over $30 \mathrm{~s}$. The other selected records also contain the EQ's and the MEQ's which preceded the EQ's (but a number of the MEQ's was less than that in the previous example). 
The MEQ's preceding the main shock were detected in the HA records by Mogi (1988) and Spindel (Spindel et al., 1974). Mogi registered the MEQ's in the near epicentral zone by one hydrophone, which was deployed in the water layer from the ship. They considered the "close" and the "remote" MEQ's as different types of the MEQ's.

The second data series was obtained in the framework of International Program ATOC (Acoustic Thermometry of the Oceanic Climate). The records registered by an HA array located near the eastern coast of Kamchatka Peninsula in the Pacific Ocean were analyzed. The array was deployed in the near bottom water layer. It was composed of several hundreds of hydrophones which were grouped in separate blocks (channels, clusters) that allowed us to derive the direction to the source from the time lags of signal arrivals in different channels.

The data contained 162 digital records obtained during the period from 12.07.1998 to 21.03.1999. The length of each hydro-acoustic record (HAR) was $1323.73 \mathrm{~s}$ (slightly above than $22 \mathrm{~min}$ ). The sampling rate was 300 per second; the instrumental band-pass filter isolated the frequency range from 40 to $110 \mathrm{~Hz}$. Every day there were up to 6 scheduled records (performed at 2:54:20, 6:54:20, 10:54:20, 14:54:20, 18:54:20, and 22:54:20 UTC). The HA records which were coincident with the seismic events or preceded them (by no more than 3-4h) were selected for the subsequent analysis. Two types of seismic acoustic signals, namely MEQ's and MRP which preceded the EQ's, were detected in the selected records.

The MEQ's occurred from several minutes up to three hours before the main shock. The signals have very sharp amplitude variations within the first 1-2 s (20 times HA signal amplitude amplification over the background level). According to our estimates, the MEQ's originate not so far under the ocean floor (the $H_{S}$ value $\leq 500 \mathrm{~m}$ ) whereas the EQ hypocenter locations may be significantly deeper (tens of kilometers). The azimuths from the HA receiver to the MEQ sources and to the epicenter of the future EQ demonstrate close agreement. In most cases, the MEQ's occur as a set of events from the closely located sources. The MEQ duration varies in the most cases from 1 to $4 \mathrm{~s}$, therefore, the Ml value of the MEQ's varies from -2.5 to -1.8 . The $F_{\max }$ value for MEQ varies from 60 to $75 \mathrm{~Hz}$. The $L_{s}$ values vary accordingly from 1 to $0.4 \mathrm{~m}$.

The MRP's with duration from $0.01 \mathrm{~s}$ to $0.05 \mathrm{~s}$. also were detected in this data series. The MRP occurred in the most cases as a train of signals. The Ml value for MRP can be estimated from -10 to -8 . These signals have also a very sharp amplitude jump. A MRP signal contains high-frequency components. But the frequency range above $110 \mathrm{~Hz}$ was inaccessible in this data series and it is therefore, impossible to estimate the linear size of the MRP sources, as well as the $H_{S}$ value in the frame of this experiment.

The typical record without seismic events and the record with EQ onset in the very end of the record are presented in
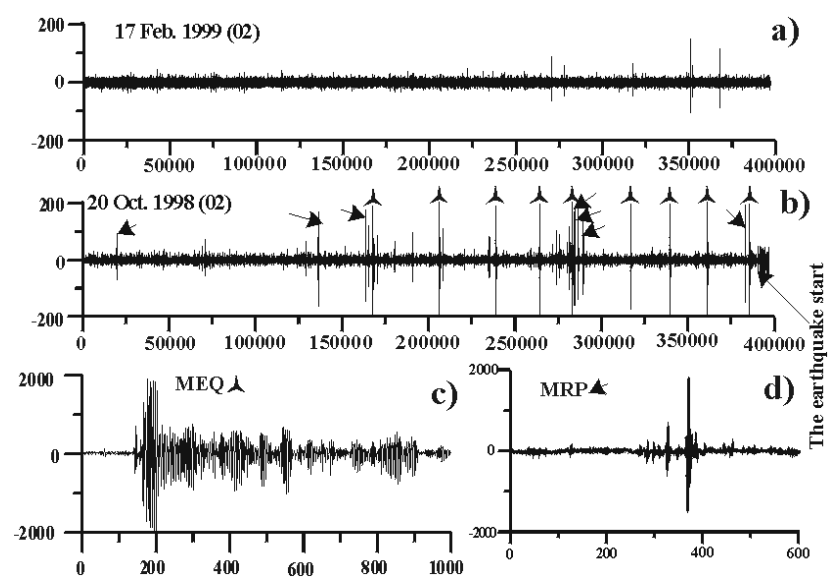

Fig. 2. Two ATOC records: the typical record without seismic events (a) and the HA record with EQ which starts in the very end of the record (b). The MEQ's are labeled as triangle star and the MRP's are identified as arrows. One MEQ with duration $3 \mathrm{~s}$ is presented on the Fig. 2c and the sequence of the MRP's is shown on the Fig. 2d. The horizontal axes for all fragments are time axes in samples (300 samples per sec.); the vertical axes for all fragments are in $\mathrm{mV}$.

Fig. 2a and $b$, respectively. The set of MEQ's and MRP's signals are depicted in Fig. 2b. The horizontal distance from the epicenter to the receiving array was $50.4 \mathrm{~km}$. The signals have very sharp amplitude variations within the first $1-2 \mathrm{~s}$ (20 times the HA signal amplitude amplification over the background level). The Fig. 2d contains a sequence of MRP's, whose frequency is estimated as $100 \mathrm{~Hz}$, and its sources are located directly under the receivers.

In those cases when the EQ started several tens of minutes after the end of the observation session, we also detected the MEQ's preceding the main shock if the time interval between the record termination and the EQ start did not exceed 3-4 h. The analysis of the second observation series was described in detail by (Morozov, 2002; Sasorova et al., 2002; Lappo et al., 2003).

The third set of HA observations analyzed in this work was obtained by Kamchatka research team (Kuptsov et al., 2005) into a water reservoir (lake) not far from the Pacific coast of Kamchatka, in the area with very high seismic activity. The observations were carried out from 2000. About 60 EQ's were registered and processed. Several HA receivers with the frequency pass band from 0 up to $10 \mathrm{kHz}$ were deployed in the water layer not far from the lake bottom.

The sharp increase of the MRP signal density in time unit was noticed 20-30 $\mathrm{h}$ before the EQ's, whose epicenters were located in the Pacific near the Kamchatka coastline. The radiation of these signals continued several hours $(10-15 \mathrm{~h}$.) and after that the density of the MRP signals vanished almost completely $10-17 \mathrm{~h}$ before the main shock. The set of MRP signals may either be chaotically distributed in time (Fig. 3b) or form a train of signals of the same type (Fig. 3a). 


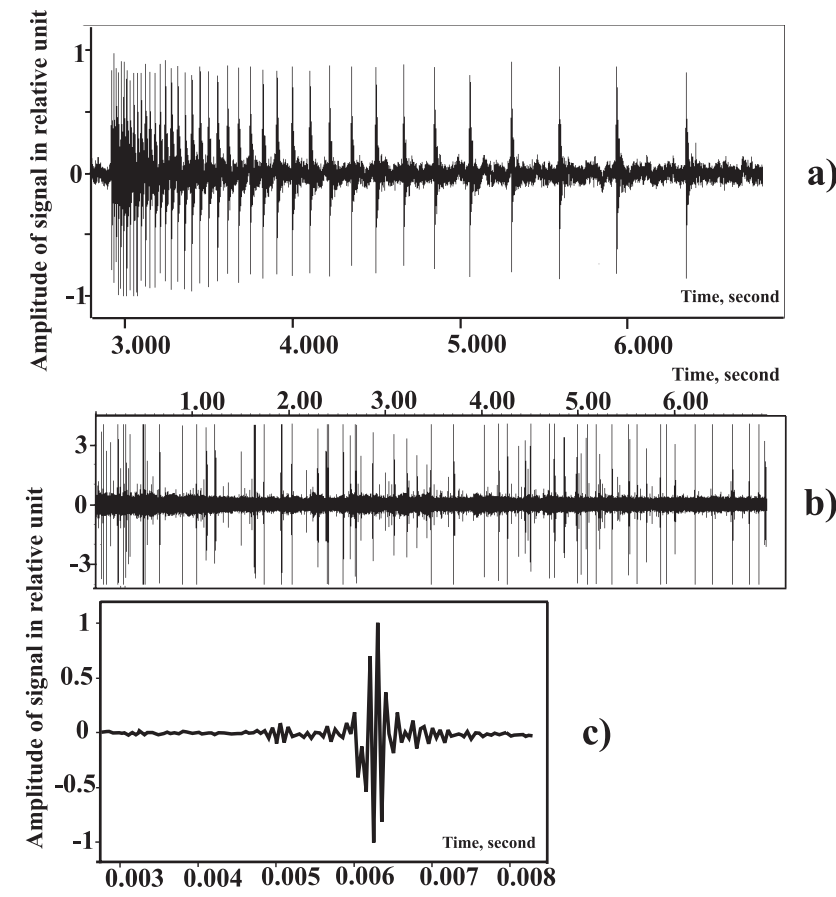

Fig. 3. Three fragments of the HA records: the typical train of signals (a), the set of MRP signals, chaotically distributed in time (b), and one signal in very detailed time scale (c). Adopted from (Kuptsov et al., 2005).

One signal from the set of signals presented in Fig. $3 b$ is shown (Fig. 3c) in more detailed time scale (the arrival time of the $P$ and the $P s$ can be determined). According to our estimates the acoustic signal sources were located not far from the receivers directly under the lake bottom (from 3 to $20 \mathrm{~m}$ ). The average value of one signal duration was about $15 \mathrm{~ms}$, thus average $\mathrm{Ml}$ value can be estimated as $\mathrm{Ml}=-8$. Acoustic signals radiated at this stage are characterized by high frequency from 1 to $6 \mathrm{kHz}$. The consistent registration was realized if the distance between EQ epicenter and HA receivers did not exceed $260 \mathrm{~km}$.

The MPR's originated from the large area (the linear size $<=520 \mathrm{~km}$ ) tens hours before the main shock, and vanished several hours before the EQ. The observed stage of the EQ preparation continues several hours. The MRP's occur in solid media permanently, but the density of these signals is small and insignificant in comparison with the MRP density in the EQ preparation stage. The MEQ's were not observed in this series of observations.

The fourth series of HA observations. On August 2006 we carried out an experiment in which we established for the first time a scheme of integrated (operative and long-term) earthquake forecast based on the hydro-acoustic and seismic data obtained during field measurements in the region of the expected event (Levin et al., 2007). While analyzing the spatiotemporal EQ distribution in the southern part of Sakhalin

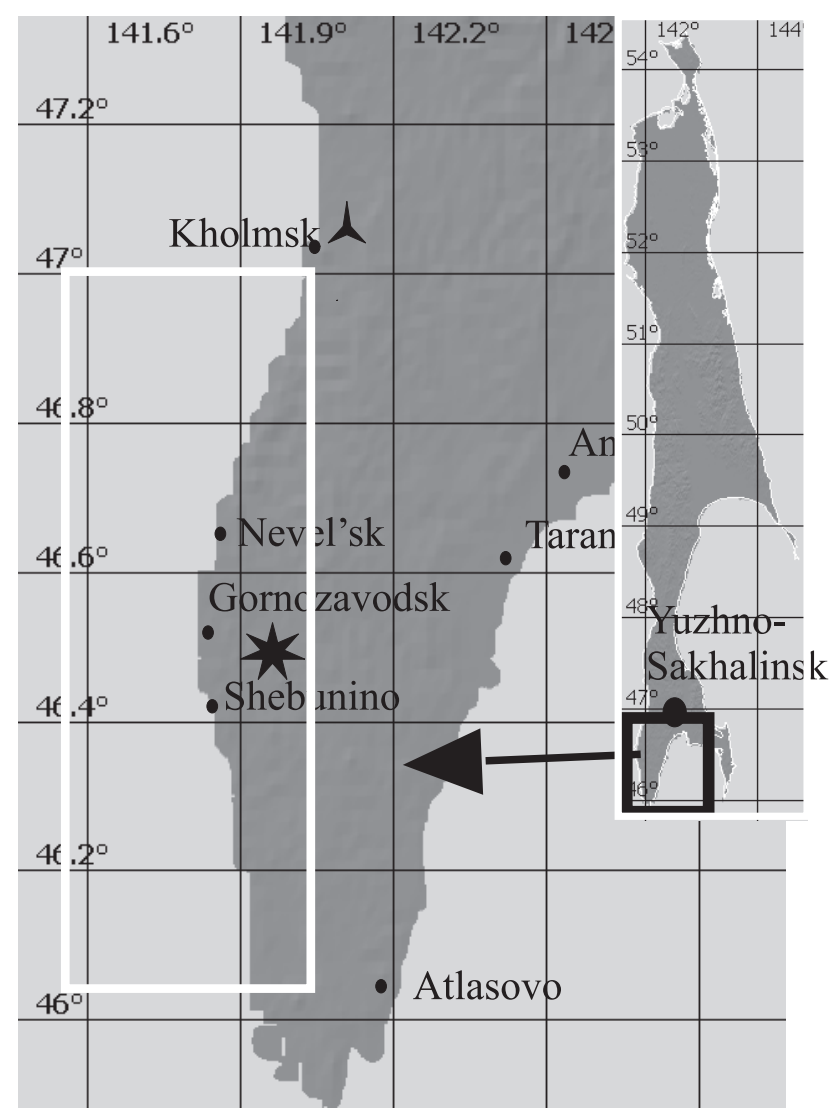

Fig. 4. Sakhalin Isl. and its southwestern part. Black seven-point star is the EQ epicenter; black three-point star is the mobile station location; bold white rectangle is the seismic gap of the first type.

in 2005 (Fig. 4), seismic gaps of type 1 (based on strong earthquakes) and type 2 (based on weak events) were found in the Kholmsk region (forecasted parameters: time - January 2006 up to July 2013; $M=6.0$ to 7.2 ; and $H=0-30 \mathrm{~km}$ ).

A mobile station for simultaneous recording of seismic and hydro-acoustic signals was deployed in a closed water reservoir near Kholmsk (coord.: 47.117 $\mathrm{N}$ and 142.096 E) in mid-August 2006 (Levin et al., 2007). All channels were synchronized. The station was located in a securely guarded zone, and the probability of generation of anthropogenic signals is insignificant. The permanent registration started on 11.08.2006.

The series of similar signals (trains) appeared in the hydroacoustic channels (Fig. 5) starting from 16.08.2006. The first train appeared at 18:57:05.5 (hereafter, UTC), i.e., 21:22:40 before the EQ start. 17 August after 05:13:04 (i.e. 11:6:03 before the main shock) the signals of this type were no longer recorded. In the subsequent records, 243 trains of similar signals were distinguished. The number of signals in the train varied in a broad range (from 7 to 130). No significant changes were observed at this time in the seismic channels. The earthquake $(\mathrm{Mb}=5.6$, coordinates of the epi- 

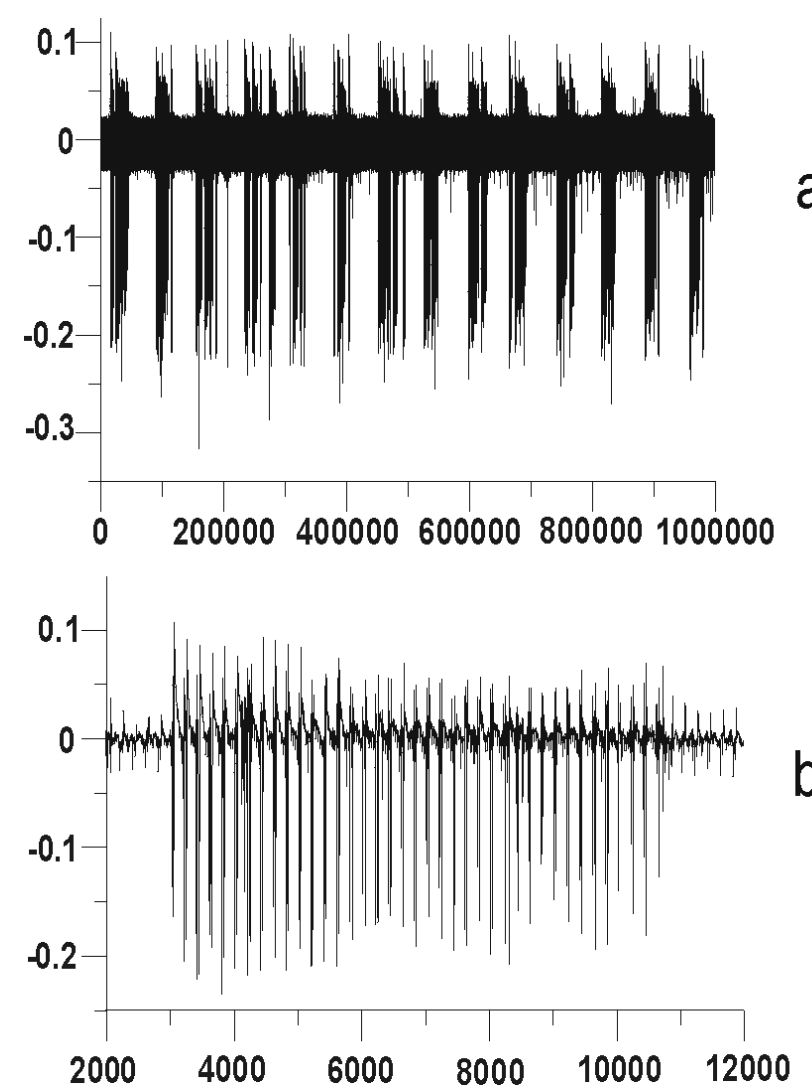

Fig. 5. Series of signal trains recorded by the HA channel of the station. The fragment (a) shows the compressed record (duration 02:53:20) with the series of signal trains. The fragment (b) shows one train of signals from the presented series (duration $50 \mathrm{~s}$ ). The number of counts is shown along the horizontal axis for all fragments (one counts is $0.005 \mathrm{~s}$ ). the amplitude of the signal is shown along the vertical axis (in $\mathrm{mV}$ ).

center $46.586 \mathrm{~N}$ and $141.857 \mathrm{E}, H=12 \mathrm{~km}$.) occurred on the 17.08.2006 and was recorded at 16:20:08 by all channels of the seismic hydro-acoustic station. The distance between the station and the EQ epicenter was $61 \mathrm{~km}$.

\section{Summary}

The sketch of the SAS evolution in the EQ preparation zone is shown in the Fig. 6. The EQ preparation area is presented for 6 moments of time from $t_{0}$ to $t_{5}$. The sequence of the SAS, which may be registered in different points of the EQ preparation area, is shown in the left part of the sketch. The SAS in point $\mathrm{A}, \mathrm{B}$, and $\mathrm{C}$ appears several tens hours before the EQ and disappears a few hours before the EQ, but the durations of the SAS in point A are significantly shorter than the signal durations in points B and C. The SAS in the epicenter area (point E) continues up to the EQ start. Both the beginning and the end of the record of the seismic-acoustic

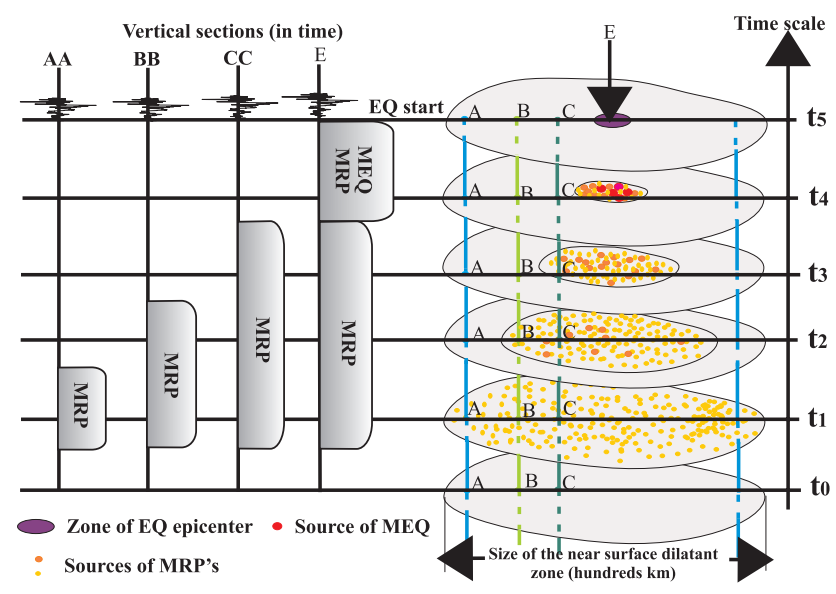

Fig. 6. The sketch of the SAS evolution in the EQ preparation zone.

signals can indicate that the earthquake preparation is in the critical stage.

Thus it was shown that the critical stage of the EQ preparation continues a few tens hours and this process has a hierarchical nature. At first time the micro-ruptures occur over a large area. Then the high frequency radiation begins to decrease, the SAS emission area begins to shrink, and the micro-earthquakes occur in the area surrounding the epicenter of the preparing EQ. The obtained results are in close agreement with theoretical conception about the appearance and the evolution of the SAS in the surface dilatant zone and results of laboratory experiments on rock sample destruction.

Acknowledgements. The research was supported by the Russian Foundation for Basic Research (Grants No. 04-05-64883-a, 06-0508098-OFI) and by the Program of Leading Scientific Schools of Russia (Grant NS-2104.2003.5). The authors thank A. V. Kuptsov, Ju. V. Marapulets, B. M. Shevtsov for observation data, assistance, useful comments and discussions.

\section{Edited by: P. Fabian}

Reviewed by: two anonymous referees

\section{References}

Alekseev, A. S., Belonosov, A. S., and Petrenko, V. E.: A concept of multidisciplinary earthquake prediction based on an integral precursor, Problems in Lithosphere Dynamics and Seismisity, Computational Seismology, Issue 32, 81-97, 2001.

Clay, C. S. and Medwin, H.: Acoustical Oceanography, J. Wiley, New York, 576 pp., 1977.

Michihiro, K., Hata, K., Fujiwara, T., Yoshioka, H., and Tanimoto, T.: Study on estimating initial stress and predicting failure on rock masses by acoustic emission, in: Rock Mechanics and Rock Physics at Great Depth, Rotterdam, 1025-1032, 1989.

Kuptsov, A. V., Larionov, I. A., and Shevtsov, B. M.: Geoacoustic emission during the precursory periods of Kamchatka earthquakes, Volcanology and Seismology, 5, 45-59, 2005. 
Lappo, S. S., Levin, B. W., Sassorova, E. V., Morozov, V. E., Didenkulov, I. N., and Karlik, Ya. S.: Hydroacoustic Location of an Oceanic Earthquake Origin Area, Dokl. Earth Sci., 389, 2, 229-232, 2003.

Levin, B. W., Sasorova, E. V., Kim, Ch. U., Korovin, M. E., Malashenko, A. E., Savochkin, P. V., and Tikhonovm, I. N.: The Sakhalin Earthquake on August 17(18), 2006, and the First Realization of Integrated Forecast, Dokl. Earth Sci., 412, 1, 117-121, 2007.

Mogi, K.: Earthquake Prediction, Academic Press, Tokyo/New York/Orlando, 355 pp., 1985.

Ponomarev, A. V., Zavyalov, A. D., Smirnov,V. B., and Lockner, D.,A.: Physical modeling of the formation and evolution of seismically active fault zones, Tectonophysics, 277, 57-81,1997.

Morozov, V. E.: High-frequency hydro acoustic signals (40$110 \mathrm{~Hz}$ ) preceding earthquakes on the Pacific shelf of the Kamchatka peninsula, Proc. of the international workshop: Local tsunami warning and mitigation 2002, 99-106, 2002.

Sassorova, E. V. and Levin, B. W.: The low frequency seismic signal foregoing a main shock as a sign of the last stage of earthquake preparation or preliminary rupture, Phys. Chem. Earth, C, 26, 10-12, 775-780, 2001

Sassorova, E. V., Didenrulov, I. N., Karlic, Yak. S., Levin, B. W., Morozov, V. E., and Petrochenko, S. P.: Near-Shore oceanic earthquakes: application of acoustic methods to detect earthquake precursory phenomena and possible improvement of tsunami warning systems, Proc. of the international workshop: Local tsunami warning and mitigation 2002, 146-158, 2002.
Sasorova, E. V., Levin, B. W., and Morozov V. E.: Local tsunami warning problem and the one of possible method of its solving, Proc. of 22-nd International Tsunami Symposium, Chania, Greece, 204-210, 2005.

Sheriff, R. E. and Geldart, L. P.: Exploration Seismology, 2nd ed., Cambr. Univ. Press, 592 pp., 1995.

Smirnov, V. A., Ponomarev, A. V., and Zavjalov, A. D.: Peculiar properties of formation and evolution of the acoustic regime structure in rock samples, Dokl. Earth Sci., 343, 6, 818-823, 1995.

Sobolev, G. A.: Essential Principles of the Earthquake Forecast, Nauka, Moscow, 314 pp., 1993.

Soloviev, S. L. and Kovachev, S. A.: The local magnitude determination for local earthquakes according to observations of the ocean-bottom seismograph, Isvestiya, Phys. Solid Earth, 5, 2630, 1996.

Spindel, R. C., Devis, S. B., Macdonald, K. C., Porter, R. P., and Phillips J. D.: Micro-earthquake survey of Median Valley of Mid-Atlantic ridge at $36^{\circ} 30^{\prime} \mathrm{N}$, Nature, 248, 577-579, 1974. 